\title{
RFID-based Hospital Real-time Patient Management System
}

\author{
Belal Chowdhury \\ School of Business, La Trobe University \\ Melbourne, Victoria 3086, Australia \\ Email: mbchowdhury@students.latrobe.edu.au
}

\author{
Rajiv Khosla \\ School of Business, La Trobe University \\ Melbourne, Victoria 3086, Australia \\ Email:r.khosla@latrobe.edu.au
}

\begin{abstract}
In a health care context, the use RFID (Radio Frequency Identification) technology can be employed for not only bringing down health care costs but also facilitate automating and streamlining patient identification processes in hospitals and use of mobile devices like PDA, smart phones, for design a health care management systems. In this paper, we outline a RFID model for designing a system in the health care. An application of the architecture is described in the area of RFID-based Real-time Hospital Patient Management System (HPMS).
\end{abstract}

\section{Introduction}

Recent years, in almost every country in the world, substantial financial resources have been allocated to the health care sector. Technological development and modern medicine practices are amongst the outstanding factors triggering this shift. Developed countries like Australia are currently facing a middleand older-aged marketplace from a predominantly youth-driven marketplace. This trend is resulting in a greater demand for health care-related services and greater competition among health care providers [1].

Achieving a high operational efficiency in the health care sector is an essential goal for organizational performance evaluation. Efficiency uses to be considered as the primary indicator of hospital performance [2]. From a managerial perspective, understanding the hospitals cost structure and their inefficiency in utilizing resources is crucial for making health care policies and budgeting decisions. The cost of medical services in hospitals is likely control by higher operational efficiency and to provide more affordable care and improved access to the public [3].

In the last few years' health care providers are making a concerted effort in using information technology for bringing down the spiraling health care cost in Australia and in the western world. In order to bring down the cost and improve efficiency, intelligent systems can play a significant role in providing intelligently processed and personalized information about patients to doctors, their health care staff (i.e., nurses) and health care administrators.

The performance of health care management system is far behind compared to the service and manufacturing industries. Health care organizations nowadays are dealing with greater rank diseases, their cost, quality and delivery has essentially not improved significantly, and even the difference with the other industries seems to have increased [1] further.

There is growing concern about maintaining one's health as population ages, mobile/sensor technology are expected to provide real-time information about vital signs and other physiological indicators of one's health and fitness. Such monitoring systems are expected to find greater use in such applications as hospitals, home health monitoring, physician's offices, elderly care facilities, fitness centers, and health research studies [12].

In a health care context, we might consider the Internet to deliver services in remote areas. Mobile commerce can also be used to automate the delivery of life saving drugs and, simultaneously, to alert emergency personnel that there might be a health problem developing [13]. So far, the Internet makes an entire range of new technologies available to hospitals to facilitate communications and build relationships [14].

The application of these principles can be facilitated by the use of the mobile technology such as Radio Frequency Identification (RFID). Today's advanced technology is capable of uniting smart tags (RFID) and data processing into a single integrated system [2]. RFID is a killer technology that elegantly provides a solution to wide range of business needs [4] including healthcare sector. It is also observed that a medical application such as RFID-based Real-time Hospital Patient Management System (HPMS) offers 
the tremendous benefits of healthcare management systems.

This paper is structured as follows: Section 2 outlines the RFID model used for developing a health care system. Section 3 illustrates the benefits of adopting RFID in health care system. Section 4 outlines the six layers of the health care systems architecture. Section 5 illustrates the application of the health care systems architecture using a RFID-based HPM. Section 6 illustrates the implementation of HPMS application. Section 7 concludes the paper.

\section{RFID Model for Healthcare Systems}

Healthcare providers (i.e., hospitals) traditionally use a paper-based 'flow chart' to capture patient information during registration time, which is updated by the on duty nurse and handed over to the incoming staff at the end of each shift. Although, the nurses spent large amount of time on updating the paperwork at the bedside of the patient, it is not always accurate, because this is handwritten.

The nurses play a vital role at the Hospital system in the success of both inpatient and outpatient care. They also play a very important role in bridging to execute clinical orders or to communicate information between the hospital and the patient that motivates to evaluate the potential of RFID (Radio Frequency Identification) technology, and to reinforce the critical job of information handover.

RFID is one of the emerging technologies offering a solution, which can facilitate automating and streamlining safe and accurate patient identification, tracking, and processing important health related information in health care sector such as hospitals [6].
Each RFID tag/wristband is identified by a Unique Identification Number (UIN) that can be programmed either automatically or manually and then password protected to ensure high security.

RFID wristband can be issued to every patient at registration, and then it can be used to identify patients during the entire hospitalization period. It can also be used to store patient important data (such as name, patient ID, drug allergies, drugs that the patient is on today, blood group, and so on) in order to dynamically inform staff before critical. RFID encoded wristband data can be read through bed linens, while patients are sleeping without disturbing them [7].

RFID technology provides a method to transmit and receive data from a patient to health service provider/medical professionals without human intervention (i.e., wireless communication). It is an automated data-capture technology that can be used to identify, track, and store patient information electronically contained on RFID wristband (i.e., smart tag). Although, medical professionals/consultants can access/update patient's record remotely via WiFi connection using mobile devices such as PDA (Personal Digital Assistant), laptops and other mobile devices. WiFi (wireless fidelity'), a wireless local area networks (WLAN) that allows healthcare provider (e.g., hospitals) to deploy a network more quickly, at lower cost, and with greater flexibility than a wired system [5].

RFID technology mainly consists of a Transponder (smart tag), a Reader and Healthcare Provider IT Systems (HPITS) as shown in Figure 1. Each tag attached to the patient wristband contains an antenna and a tiny microchip smaller than a grain of sand.

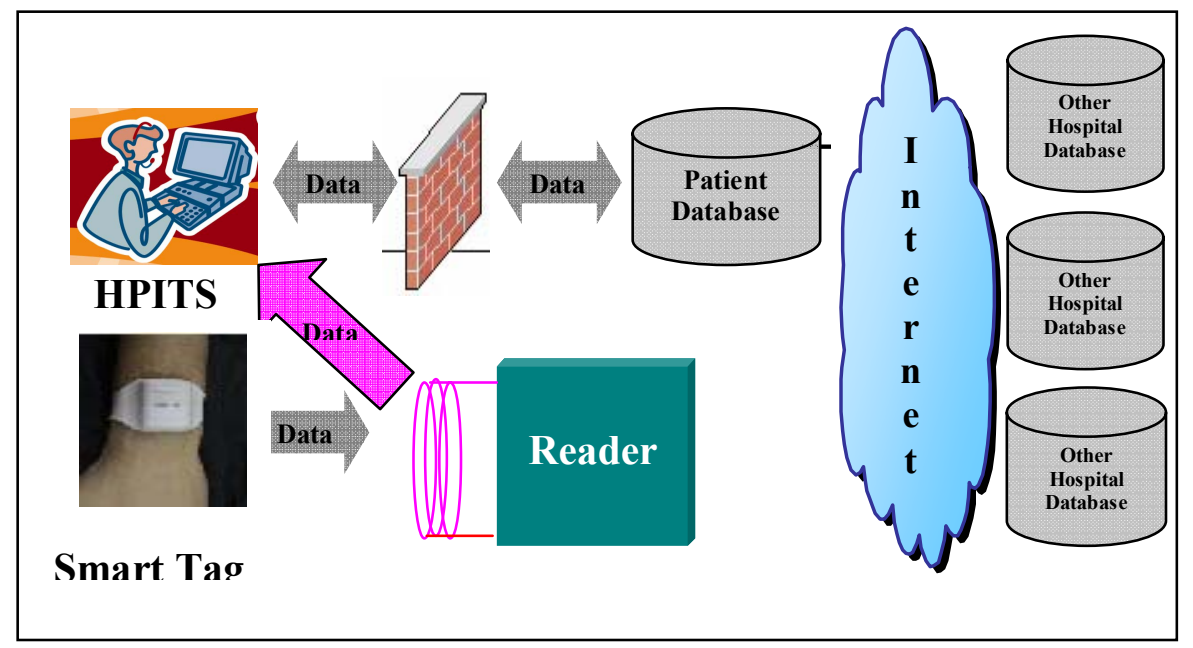

Figure 1: Main components of RFID-based patient management system 
The antenna picks up radio-waves or electromagnetic energy beamed at it from a reader device and enables the chip to transmit patients unique ID to the reader device, allowing the patient to be remotely identified. The reader converts the radio waves reflected back from the patient wristband into digital information that can then be passed onto HPITS for processing.

Patient's basic important data (e.g., patient ID, name, age, location, drug allergies, blood group, drugs that the patient is on today) can be stored in the patient's back-end databases for processing. Patient databases can also be linked through Internet into other hospitals databases [15].
Potential benefits such as improve patients safety, eliminates paper-based document (e.g., bed side card), cost savings, increases efficiency and productivity, prevent/reduce medical errors, reduce patient waiting time, and so on, of using RFID technology within health care sector (e.g., hospitals) is numerous as shown in the Figure 2.

\section{Benefits of adopting RFID-based Healthcare System}

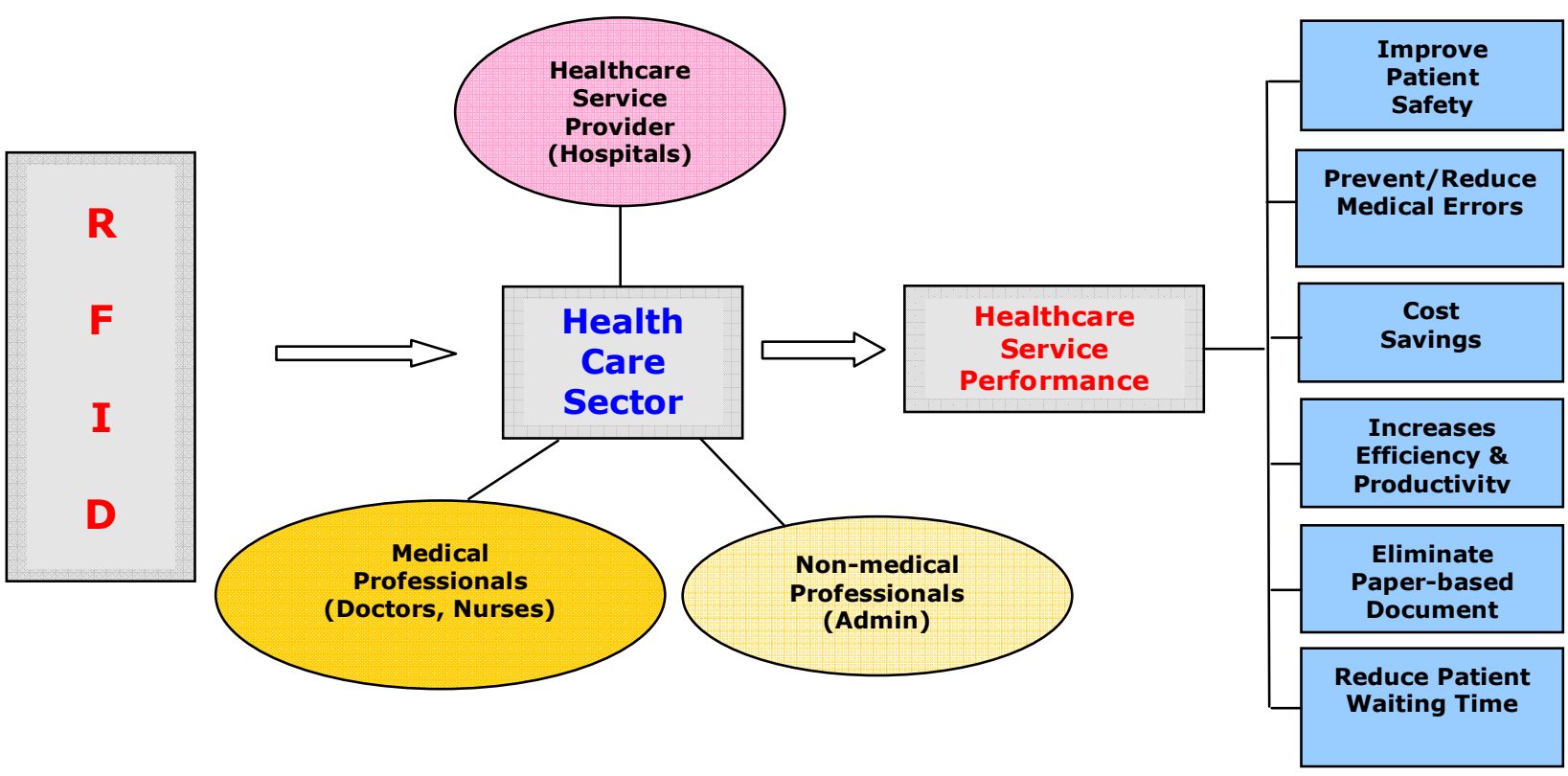

Figure 2: Benefits of using RFID in healthcare sector

\section{Multi-layer Architecture for Healthcare Systems}

Figure 3 shows the multi-layered (i.e., six layer) RFID-based healthcare systems architecture, namely, physical layer, middleware layer, process layer, data access layer, application layer and user interface layer.

The physical layer consists of the actual hardware components that include RFID tag, antennas and readers.

RFID Middleware is the interface between the RFID reader and healthcare providers (i.e., hospitals) databases and patient management system. It is an important element of RFID systems in healthcare sector. RFID middleware layer enables healthcare providers (e.g., hospitals) a quick connectivity with readers, lower the volume of information that medical applications need to process by grouping and filtering raw RFID observations from readers, and provide application-level interface for managing readers and process large volumes of RFID data for their medical applications. This layer is also monitoring physical layer components and supports International Organization for Standardization (ISO) standard [8].

The Process Layer drives hospitals to deploy RFIDbased healthcare system (business) processes that 
provide real-time integration into existing systems. This layer enables data mapping, formatting, business rule execution and the service interactions with databases. The data access layer composed of a Relational Database Management System (i.e., normalization, database schema) and applications that allow healthcare providers for the creation of RFID "events". This layer includes a data loading approach that supports high volumes of RFID data, access them using structured query Language (SQL) and enables the data to be easily presented through the use of customized views to healthcare provider (i.e., hospital) to use them [9].

The application layer interacts with multiple patients' wristband in the medical application (i.e., patient management system). Finally, the user interface layer is comprised of an extensible, graphical user interfaces that allow RFID devices (e.g., reader, RFID tag) in a uniform, user-friendly way to work seamlessly in a Windows environment.

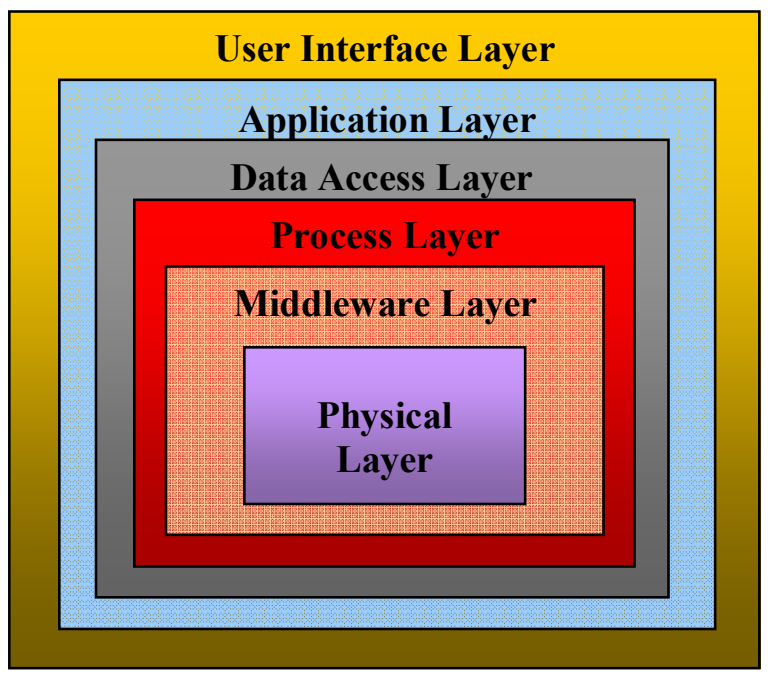

Figure 3: Multi-layer architecture for health care systems

\section{RFID-based Real-time Hospital Patient Management System (HPMS) Application}

Figure 4 shows the GUI (Graphical User Interface) based HPMS application developed by Agile System Development Methodology (ASDM) using C\# in Microsoft Visual Studio.net 2003 environment. ASDM enables rapid software development methods that are faster, cheaper, and more responsive to user needs than the traditional (e.g., SDM - Software Development Methodology) of software engineering [10]. The SDM (e.g., waterfall methodology) provides an orderly sequence of development steps that being needlessly slow and cumbersome to ensure the quality, reliability, and maintainability of the developed software. While agile method places more emphasis on people, working software, customer collaboration and responding to changes rather than processes of software development (i.e., plans, contracts and tools) as an orderly, rational process. ASDM recommends activities that are often context dependant and a variety of practices for constant feedback on customer requirements, and technical decisions [11].

The HPMS uses the unique ID transmitted by RFID tags as a key to information stored in the HPITS backend databases. For example, an RFID tag attached to a patient wristband only contains an RFID tag ID, which an application can use to retrieve patient record stored in the hospital databases. When a RFID reader reads multiple patient wristbands that have embedded RFID tags, the application lists and displays all ID's in patient info area. Click on a particular ID will display the information for that patient.

In addition, healthcare professionals (e.g., doctors, consultants) can edit/update password protected patient's medical record for increased patient and data security by clicking the Update Patient Button. 

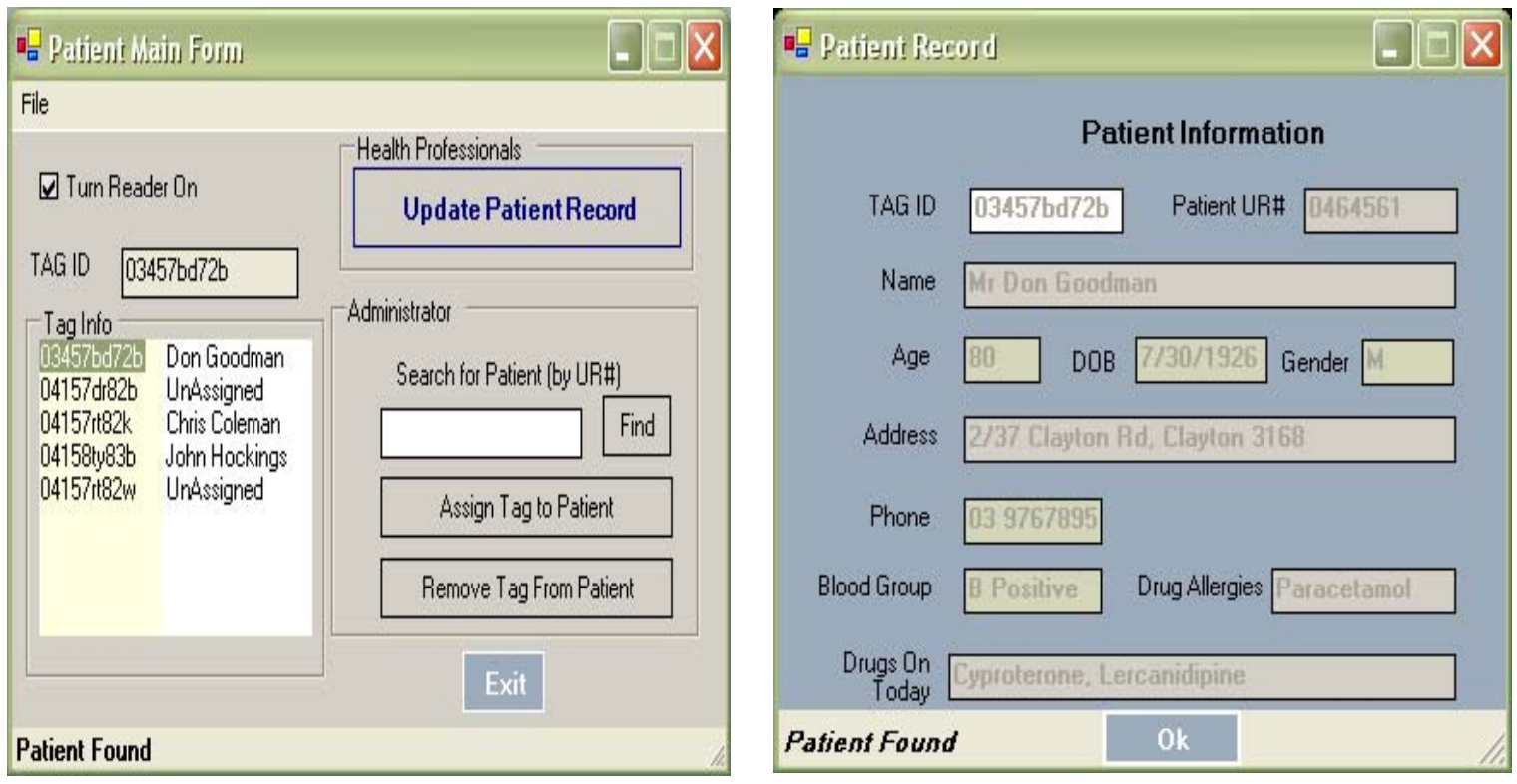

Figure 4. RFID-based HPMS

The Patient Management System's administrator can issue unused tag (wristband) to every patient at registration time by using the 'Assign Tag to Patient' button at the bottom part (i.e., administrator's section) of the application. This wristband can be used to identify patients during the entire hospitalization period. The tag (wristband) can be removed from the patient using 'Remove Tag from Patient' button on discharge from the hospital. As RFID tags are reusable, the available tag can be assigned to a new patient.

\section{Implementation of Hospital Patient Management Systems}

The final version of HPMS application can be implemented in departments (e.g., medicine, surgery, obstetrics and gynaecology, paediatrics) in both public and private hospitals for fast and accurate patient identification without human intervention. But, most of the patients at public hospitals get admission into different units (e.g., medicine) through emergency department. So, our immediate focus on hospitals emergency department to implement HPMS. At present, we are in the process of discussion with emergency department in one of the busiest hospital in Melbourne, Australia to install RFID-based HPMS.

\section{Conclusions and Future Work}

In this paper we have described multi-layer health care system architecture for design of RFID-based Hospital Patient Management System. We have shown the application and implementation of the above system. Using HPMS, health care providers (e.g., hospitals) have a chance to track fast and accurate patient identification, improve patient's safety by capturing basic data (such as patient unique ID, name, blood group, drug allergies, drugs that the patient is on today), prevent/reduce medical errors, increases efficiency and productivity, and cost savings through wireless communication. The HPMS also helps hospitals to build a better, more collaborative environment between different departments, such as the wards, medication, examination, and payment.

This generic architecture of health care system can be applied to other areas such as new born babies and aged-care management in both public and private hospitals. Where, health care providers (i.e., hospitals) will have an opportunity of use smart tag (RFID) for people's babies, which look pretty much alike to ensure that they match the right mother with the right baby. Having RFID tracking means hospitals can use RFID-based HPMS to track whether infants are even inside the hospital that presumably is useful if they lose track of patients. The similar concept can be applied for the aged-care management using RFID-based HPMS. 
In a future version of RFID-based HPMS, we will explore the functionality to access patient's medical record from other health care providers (e.g., hospitals) databases through Internet. Finally, we are in the process of developing the functionality for accessing or updating patient records remotely by medical professionals/consultants using mobile devices like PDA/Smart phone.

\section{References}

[1] F. A. Correa, M.J. Gil, and L.B. Redín, "Benefits of Connecting RFID and Lean Principles in Health Care", Working Paper 05-4, Business Economics Series 10, 2005.

[2] P.F. Drucker, "The essential Drucker: selections from the management works of Peter F. Drucker", New York: HarperBusiness, 2001.

[3] B. Hollingsworth, P.J. Dawson, and N. .Maniadakis, "Efficiency measurement of health care: A review of non-parametric methods and applications", Health Care Management Science, Volume 2, No. 3, 1999, pp. 161-172.

[4] S. Shepard, "RFID Radio Frequency Identification", The McGraw-Hall Companies, Inc. USA, 2005.

[5] Denis Laverty, "What is WiFi? An Introduction to Wireless Networks for the Small/Medium Enterprise (SME)", http://www.openxtra.co.uk/articles/wifiintroduction.php accessed on 10 February 2007.

[6] LandMARC, "Integrated Sensor Radio Frequency Identification (ISRFID)", Georgia Tech Research Institute Electro-Optics, Environment, and Materials Laboratory - GTRI Baker Building Room 328 Atlanta, GA 30332-0834, 2002.

[7] GAO RFID, "RFID Solutions for Healthcare Industry", http://healthcare.gaorfid.com/, accessed on 10 February 2007.

[8] B. Glover and H. Bhatt, "RFID Essentials", O'Reilly Media, Inc. 1005 Gravenstein Highway North, Sebastopol, CA 95472, Jan. 2006, pp. 54169.

[9] Sybase, Inc., "SYBASE RADIO FREQUENCY IDENTIFICATION (RFID) TECHNOLOGY ARCHITECTURE“One Sybase Drive Dublin CA, 94568 USA, 2005,

http://www.sybase.com/sb content/1031464/1605 6 RFID_Arch_L02607 FNL3.pdf accessed on 11 February 2007.
[10] Kent Beck, and Martin Fowler, "Planning Extreme Programming", Addison-Wesley Professional.

75 Arlington Street, Suite 300, Boston, MA 02116, 2001.

[11] P. Abrahamsson, O. Sallo, J. Ronkainen and J. Warsta, "Agile Software Development Methods Review \& Analysis", VTT publications 478, VTT technical Research Centre of Finland, Finland, 2002.

[12] Adrian P. "Sensors Have Expanding Opportunities for Use in Wearable Devices for Health Monitoring", 2005, http://www.sensorsmag.com/sta/sta1105.shtml accessed on 25 September 2006.

[13] R.T. Watson, L.F. Pitt, P. Berthon, and G.M. Zinkhan, (2002), "U-Commerce: Extending the Universe of Marketing," Journal of the Academy of Marketing Science, Vol. 30 No. 4, 2002, pp. 329-343.

[14] T. S. Gruca and D. S. Wakefield, "Hospital web sites: Promise and progress", Journal of Business Research, Vol. 57 No. 9, 2004, pp. 1021-1025.

[15] U.S. Government Accountability Office, "Radio Frequency Identification Technology in the Federal Government", 441 G Street NW, Room LM Washington, D.C. 20548, 2005. 